\title{
CHARACTERISTICS OF ELM ACTIVITY AND FUELING EFFICIENCY OF PELLET INJECTION FROM DIFFERENT LOCATIONS ON DIII-D
}

\author{
by \\ L.R. BAYLOR, ${ }^{,}$T.C. JERNIGAN, R.J. COLCHIN, ${ }^{*}$ \\ J.R. FERRON, M.R. WADE*
}

This is a preprint of a paper to be presented at the 14th International Conference on Plasma Surface Interactions, May 22-26, 2000, Rosenheim, Germany and to be published in the Proceedings.

*Oak Ridge National Laboratory, Oak Ridge, Tennessee.

\author{
Work supported by \\ the U.S. Department of Energy \\ under Contracts Nos. DE-AC03-99ER54463 \\ and DE-AC05-000R22725
}

GA PROJECT 30033

JUNE 2000 


\section{DISCLAIMER}

This report was prepared as an account of work sponsored by an agency of the United States Government. Neither the United States Government nor any agency thereof, nor any of their employees, make any warranty, express or implied, or assumes any legal liability or responsibility for the accuracy, completeness, or usefulness of any information, apparatus, product, or process disclosed, or represents that its use would not infringe privately owned rights. Reference herein to any specific commercial product, process, or service by trade name, trademark, manufacturer, or otherwise does not necessarily constitute or imply its endorsement, recommendation, or favoring by the United States Government or any agency thereof. The views and opinions of authors expressed herein do not necessarily state or reflect those of the United States Government or any agency thereof. 


\section{DISCLAIMER}

Portions of this document may be illegible in electronic image products. Images are produced from the best available original document. 


\begin{abstract}
Pellet injection from several different locations has been used on the DIII-D tokamak to study core fueling and transport in $\mathrm{H}$-mode and $\mathrm{L}$-mode plasmas. These experiments have provided a variety of conditions in which to examine the fueling efficiency and edge localized mode (ELM) interaction with pellets injected into DIII-D plasmas. The fueling efficiency, defined as the total increase in number of plasma electrons divided by the number of pellet fuel atoms, is determined by measurements of density profiles before and just after pellet injection. New injection ports on the DIII-D inner wall enable high field side (HFS) pellet injection from both the midplane and $30 \mathrm{~cm}$ above the midplane. These ports, in addition to the previously existing top vertical ports and outside midplane port, enable a comparison of the effect of injection location on fueling efficiency and ELM activity. We find that the ELMs triggered from HFS injected pellets and vertical HFS injected pellets are similar to the nominal background ELMs in ELMing H-mode plasmas. In some cases, ELMs are not triggered directly by the HFS-injected pellets, but appear up to $50 \mathrm{~ms}$ later when the edge pressure gradient reaches the stability limit. In contrast, the low field side (LFS) injected pellets trigger large-magnitude, long duration ELMs that lead to a significant reduction in fueling efficiency. The fueling efficiency of the HFS-injected pellets is found to be significantly higher than with the LFS-injected pellets and remains high even with significant heating power in ELMing H-mode plasmas.
\end{abstract}




\section{INTRODUCTION}

Fueling a fusion grade plasma with the injection of frozen pellets of hydrogenic isotopes is an important technique developed and refined in the past 20 years [1]. In the past few years, the research in this area has concentrated on pellet fueling from the high field side (HFS), which has been shown to lead to deeper more efficient fueling of tokamak plasmas than the previously standard injection location from the outside midplane or low field side (LFS) injection [2,3]. The issue of pellet fueling efficiency has not been extensively examined under various conditions until recent studies for LFS injection [4,5] from several devices. The efficiency of the fueling system is an important parameter in developing a reactor device that can achieve minimal tritium throughputs and wall inventory. In this study on DIII-D, we examine the extensive experimental results for pellet fueling efficiency from different injection locations in one device (an elongated, diverted, tokamak plasma) under various operating conditions.

The pellet injector on DIII-D is a three-barrel pneumatic pellet injection system [6]. Deuterium pellets are produced of $2.7 \mathrm{~mm}$ diameter and length $\left(6 \times 10^{20}\right.$ atoms $)$ with speeds from 100-1000 m/s. Diagnostics in the pellet injection line measure pellet speed and mass for each pellet. The ablation process is monitored with a photodiode that observes the light emitted by the ablating pellet. The termination of the light from the photodiode and the measured pellet speed gives the penetration depth of the pellet, which has been found to differ significantly from the depth where the fuel particles are deposited. Each of three independent repeating pneumatic guns has an independent guide tube. Curved guide tubes have been installed to connect the three barrels of the injector to vertical ports ( $\mathrm{V}+1$ and $\mathrm{V}+3$ ) and to two inner wall locations (HFS 45 and HFS mid) as shown in Fig. 1. Tests of a mockup of these injection lines indicate that deuterium pellets survive intact with speeds up to $300 \mathrm{~m} / \mathrm{s}$ through the inner wall guide tubes and $500 \mathrm{~m} / \mathrm{s}$ through the vertical guide tube path. The pellets lose approximately $20 \%$ of their mass during the transport through the 12-m long guide tubes [7]. Two of the guns have been modified with a mechanical punch that is required to produce slow pellets, $<300 \mathrm{~m} / \mathrm{s}$, for injection from the inner wall locations.

The fueling efficiency, which is defined as the total increase in number of plasma electrons divided by the number of fuel atoms in the pellet, is determined by Thomson scattering measurements of electron density profiles before and just after pellet injection in conjunction with the pellet mass measured in a resonant microwave cavity. These density profile measurements have been made as close as $20 \mu$ s after completion of the pellet ablation process but are more typically made $1-5 \mathrm{~ms}$ after injection of the pellet. 
The HFS inner wall and vertical injected pellets do not traverse through the microwave cavity and so the nominal pellet size measured by injection on the outside midplane through the cavity is used in the fueling efficiency calculation. The mass lost in traversing the curved guide tubes is factored into the fueling efficiency calculation.

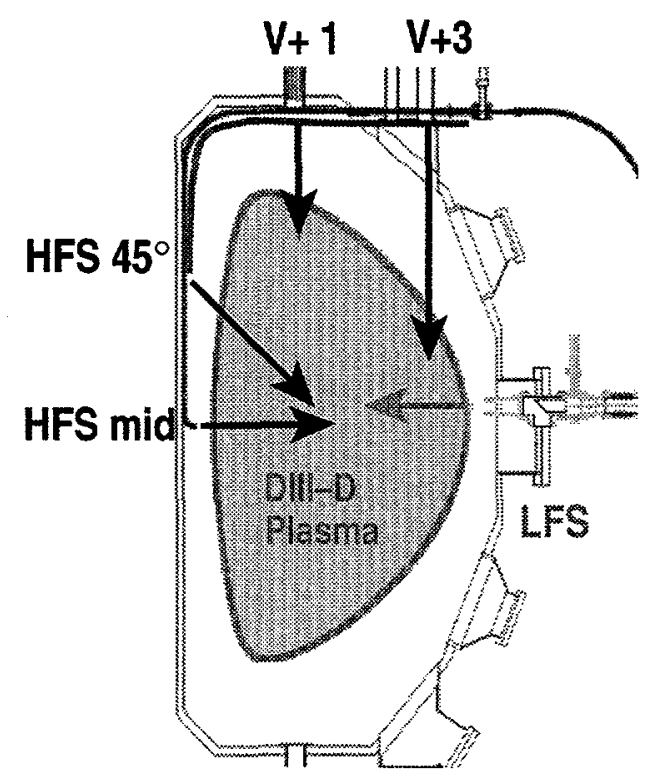

Fig. 1. The pellet injection locations on DIII-D shown in a poloidal cross section. 


\section{PELLET PLASMA INTERACTION AND ELMS}

Three effects of the plasma on the pellet can reduce pellet fueling efficiency from the ideal $100 \%$ value. Some ablation of the pellet occurs in the scrapeoff layer (SOL) before it reaches the last closed flux surface or separatrix of the plasma. This is due to energetic particles in the SOL that impinge on the pellet as it traverses the SOL. The magnitude of this ablation is rather small on DIII-D for all the injection locations as measured by $\mathrm{D}_{\alpha}$ light emitted by the ablating pellet. Another effect that can reduce the ideal fueling efficiency is the expulsion of pellet ablatant from the plasma by drift effects on the ablatant as it propagates away from the pellet along field lines. Such drifts can be caused by an $\mathrm{E} \times \mathrm{B}$ force that arises from a polarization of the ablatant cloud or from a pressure gradient driven effect on the ablatant $[2,8]$. The time scale of such drifts for DIII-D is on the order of $10 \mu \mathrm{s}$. The ablatant would be expected to move in the $-\nabla \mathrm{B}$ (outward major radius) direction and actually leave the plasma confinement region for LFS-injected pellets, thus reducing the fueling efficiency. In pellet experiments where the pellets were injected from the HFS, specifically, the inner wall $[2,3]$, the ablatant is not ejected from the confinement region. The third effect that reduces the ideal fueling efficiency is that of pellets triggering edge localized modes (ELMs) in $\mathrm{H}$-mode plasmas, which then eject particles and energy from the edge barrier plasma.

Pellets injected into $\mathrm{H}$-mode plasmas from the different locations often induce an ELM-like event $[3,9]$ that has a similar duration and magnitude of divertor $D_{\alpha}$ light perturbation and similar power incident on the divertor to a normal nonpellet-induced ELM. This suggests that pellet fueling of reactor plasmas may be no more detrimental to divertor operation than any inherent ELM activity. The ELM-like event is found to expel a significant fraction of the pellet deposited mass by inducing strongly increased particle transport at the plasma edge. The plasma transitions to L-mode following the pellet injection for a short period ( $<25 \mathrm{~ms}$ ), which is believed to be responsible for a continued expulsion of the pellet deposited particles, leading in some cases to retention of less than $20 \%$ of the pellet mass [5].

Some significant differences in the ELMs triggered from the different pellet injection locations are observed. Pellets injected from the LFS (outside midplane and V+3 ports) into previously ELMing plasmas can cause an increase in the Type I ELM frequency as shown in Fig. 2. LFS-injected pellets also trigger Type I ELMs when injected into otherwise ELM-free conditions [10] such as VH-mode and can cause synching of ELMs to the pellets when multiple pellets are injected. Compound ELMs occur when purposely shattered pellets from the LFS are injected into high power $\mathrm{VH}$-mode plasmas to trigger 
edge perturbations. Presumably, the pellets cause a rapid change in the edge pressure gradient that leads to a change in the stability against the edge localized mode. Fast profile diagnostics that can track such a fast pressure gradient response to the pellets are not yet available.

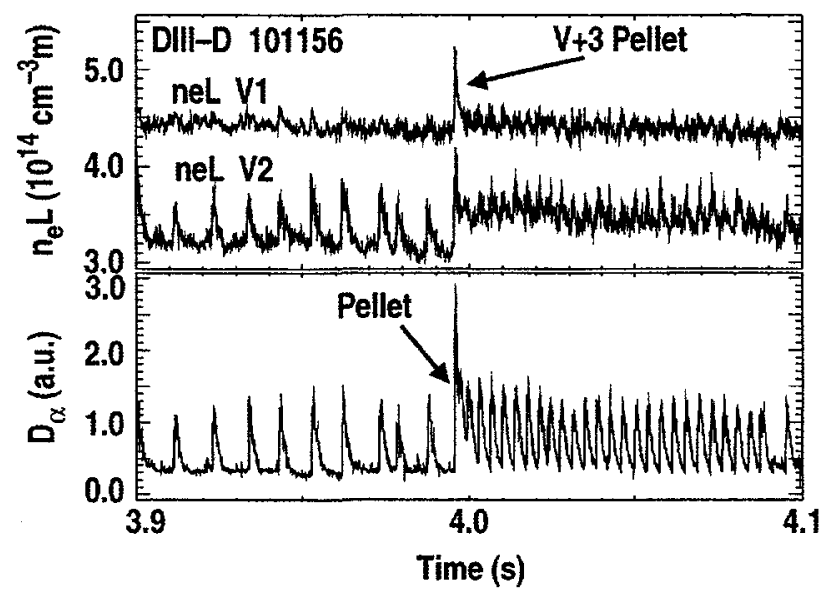

Fig. 2. The line integral density for a central and edge interferometer chord and divertor $\mathrm{D}_{\alpha}$ emission in an ELMing $\mathrm{H}$-mode plasma with $7 \mathrm{MW}$ NBI. A $2.7 \mathrm{~mm}$ pellet is injected from the $\mathrm{V}+3$ port just before $4.0 \mathrm{~s}$. The pellet induces a large ELM and increases the subsequent ELM frequency.

Pellets injected from the HFS also induce Type I ELMs in ELMing plasmas, but the divertor $D_{\alpha}$ emission after these ELMs is of much shorter duration than for LFS-injected pellets in the same discharges [3]. Triggering of ELMs is also observed when deep penetrating pellets are injected from the HFS into ELM-free VH-mode plasmas. The ELM is sometimes delayed up to $50 \mathrm{~ms}$ after the HFS pellet is injected [10], possibly resulting from the time necessary for the injected pellet particles to transport back out to the plasma edge. In an effort to reduce the usual strong ELM that terminates the VH-mode [11], both HFS and LFS-injected pellets were employed to begin an ELMing phase in VH-mode plasmas before natural ELMs occur. This scheme was successful in starting an ELMing phase earlier in the VH-mode plasmas, but did not extend the duration of the VH-mode or reduce the severity of ELMs on core plasma energy confinement.

The duration of the enhanced divertor $D_{\alpha}$ emission after ELMs triggered by pellet injection is a function of the injection location as shown in Fig. 3. Using the same collimated view of the divertor, we see that the full width at half maximum (FWHM) of the divertor light perturbation following the pellet-induced ELM is much shorter for the HFS pellets than for LFS or $\mathrm{V}+3$ pellets. This indicates there is a much larger particle flux to the divertor following a LFS pellet-induced ELM. The enhanced duration of the 
divertor perturbation for LFS pellets is believed to be caused by a larger expulsion of particles both from a stronger ELM collapse of the edge barrier and from a rapid outward major radius drift of pellet mass.

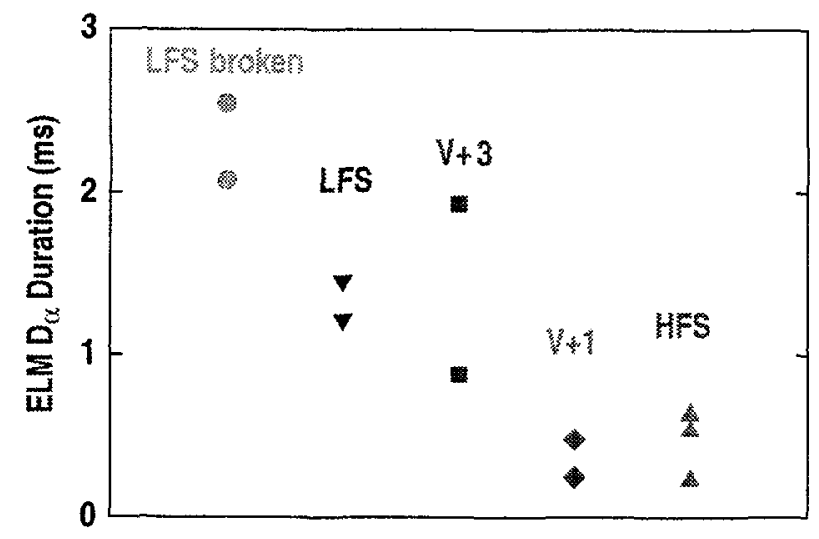

Fig. 3. The duration of the $D_{\alpha}$ emission following a pellet induced ELM for ELMs triggered by pellets injected from different locations. The full width at half the maximum value is used as the duration. The LFS broken and V+3 pellets were in an upper single-null configuration while the other cases were in a lower single null. 


\section{PELLET FUELING EFFICIENCY}

The fueling efficiency $\eta$, is defined as $\eta=\Delta N_{e} / N_{p}$, where $N_{p}$ is the pellet particle content derived from the mass measured by the microwave cavity and $\Delta \mathrm{N}_{\mathrm{e}}$ is the increase in plasma particle content determined by integrating the difference in measured Thomson scattering electron density profiles. The uncertainty in the mass measurement is typically $\pm 15 \%$ while the uncertainty in the number of plasma particles is on the order $\pm 5 \%$ for the profiles measured within $3 \mathrm{~ms}$ of the pellet event. Plots of the calculated fueling efficiency $\eta$ as a function of the neutral beam injection (NBI) power on DIII-D for the different injection locations are shown in Fig. 4. There is clearly a strong dependence of $\eta$ on the injection location. The fueling efficiency is not shown as a function of penetration depth since most of the HFS pellet data does not have very much variation in penetration depth. The fueling efficiency was shown earlier to be a strong function of penetration depth for LFS-injected pellets [5].

The pellet fueling efficiency in $\mathrm{H}$-mode plasmas is in general lower than in $\mathrm{L}$-mode for the same power and or penetration depth, especially for the LFS-injected pellets. This is presumably because of the expulsion of some of the pellet mass due to the triggering of an ELM-like event as discussed in the previous section. A significant fraction of the pellet mass is seen to be lost in the edge pedestal region, which collapses while the pellet is injected. In ELM-free $\mathrm{H}$-mode discharges, the fueling efficiency is nearly as high as in $\mathrm{L}$-mode discharges except in some cases where the pellet induced a very strong ELM.

The inner wall and vertical HFS-injected pellets in H-mode do trigger an ELM-like event; however, it appears to be smaller in magnitude than those from pellets injected on the LFS midplane or vertical port. The measured $D_{\alpha}$ light perturbation on both the lower and upper divertor locations is smaller for the HFS-injected pellets, presumably indicating a reduced expulsion of pellet mass. This reduction in $D_{\alpha}$ magnitude is believed to be due to a reduced ELM amplitude and a reduced or nonexistent ejection of the ablatant out of the core plasma, leading to improved fueling efficiency for the HFS pellets. The fueling efficiency of the vertical injected $\mathrm{V}+1$ pellets is better than outside midplane injected pellets into the same plasma discharges. The scaling of vertical pellet fueling efficiency with heating power is shown in Fig. 4(b). The deposition from these pellets is deeper than from equivalent LFS-injected pellets and is more comparable to the theoretical predictions from the NGS ablation models [12]. The HFS inner wall pellets have very deep mass deposition [3] and have fueling efficiency on the order of $90 \%$ and show no degradation with increased NBI power as shown in Fig. 4(c). Since the nominal 
pellet mass is assumed for these pellets (both inner wall and vertical), the actual mass may be lower giving a fueling efficiency that is nearly ideal.
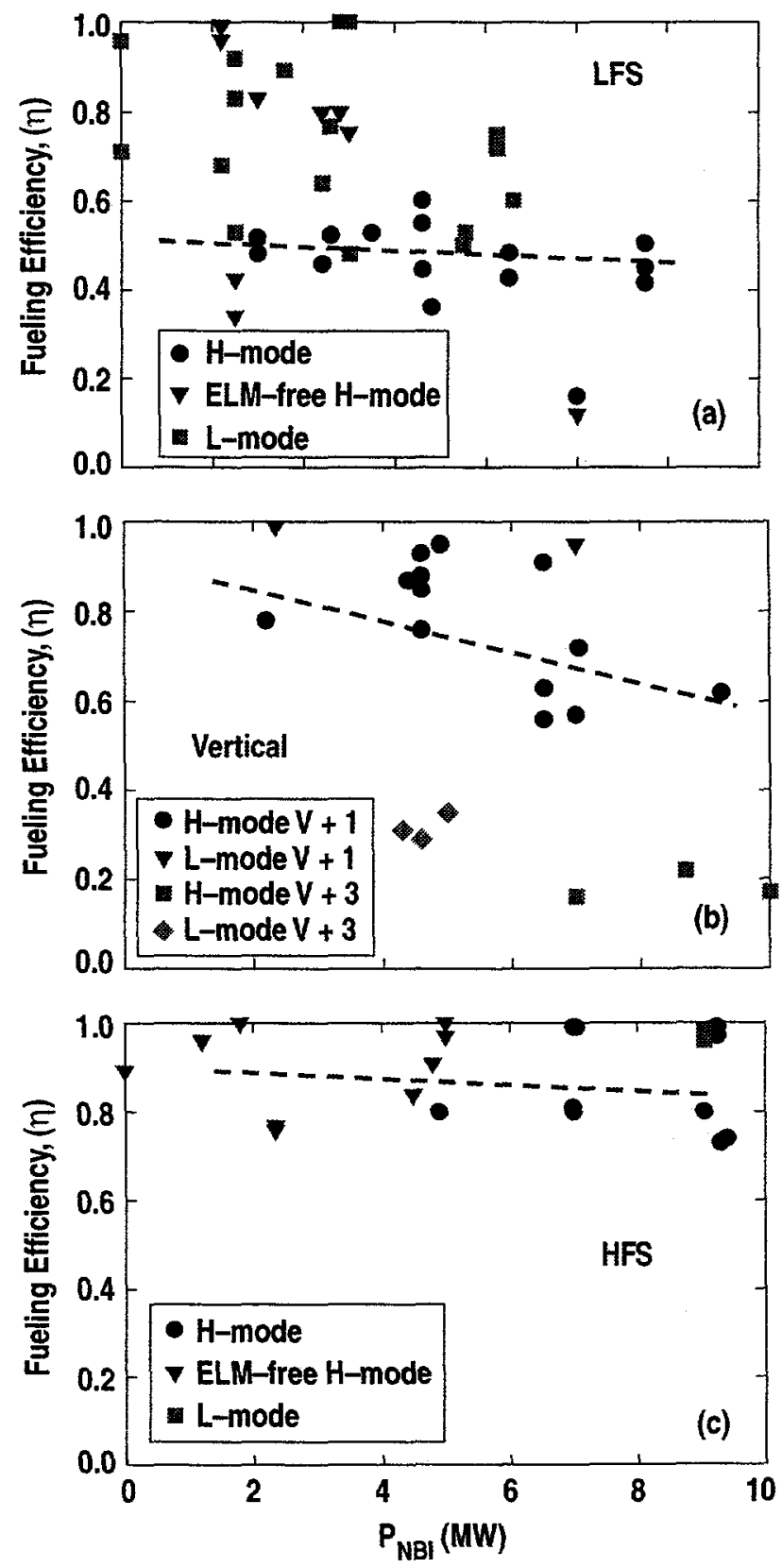

Fig. 4. Fueling efficiency as a function of neutral beam input power for (a) LFS pellets, (b) vertical pellets, and (c) HFS inner wall injected pellets. 


\section{DISCUSSION}

Injection of pellets from all injection locations on DIII-D into $\mathrm{H}$-mode plasmas has been observed to trigger ELM events, which may prove to be beneficial for situations where ELMs are needed to flush impurities or reduce edge pressure gradients. The ELMing region in the DIII-D H-mode discharges studied is in the range of $5 \mathrm{~cm}$ inside the separatrix. In all the cases on DIII-D, the pellets penetrate well beyond the ELMing region because of the pellet size and speed. If the pellets were to not penetrate beyond the ELMing region, it is likely that the fueling efficiency would be reduced. The ASDEXUpgrade results indicate a lower fueling efficiency, which may be due in part to shallower penetration that is just beyond the ELMing region in that device [9].

As has been observed on other individual machines, the pellet fueling efficiency of LFS-injected pellets is reduced when the applied auxiliary heating power is increased. In DIII-D, this correlates with higher edge electron temperatures, which enhances the pellet ablation rate in the edge region since the ablation is a strong function of the local electron temperature. For high fueling efficiency from the LFS, maximum penetration is desirable.

Magnetic field curvature and $\nabla \mathrm{B}$ in a tokamak lead to a radial outward drift of the ablatant during the process of the pellet mass symmetrization along the field lines. This drift of ablatant in the $-\nabla B$ direction pushes some of the pellet mass out of the confinement region and reduces the fueling efficiency for LFS injection. This is the motivation for attempting injection inside the magnetic axis from the inner wall and from a vertical port. The results from vertical injection of pellets inside the magnetic axis on DIII-D looks promising for increased penetration and fueling efficiency. A reduced ELM perturbation from the vertical HFS pellets is observed that is believed to be partially responsible for the improved fueling efficiency. The obvious advantage of vertical HFS injection over that from the inner wall is that an injector can be installed above the device with a straight guide tube so that high speed pellets can be injected deep into a large plasma. Inner wall injection will always limit the pellet speed, which may limit the fueling capability in a large reactor scale device.

In conclusion, pellet fueling experiments on DIII-D have been used to examine the pellet fueling efficiency from different injection locations. LFS pellet injection in ELMing $\mathrm{H}$-mode plasmas has lower fueling efficiency than in ELM-free $\mathrm{H}$-mode or L-mode plasmas due to the pellet inducing an ELM that ejects a sizeable portion of the edge density pedestal. HFS pellet injection has much higher fueling efficiency than LFS injection due to deeper mass penetration and a reduced ELM perturbation. The exciting new methods of vertical and inner wall pellet injection inside the magnetic axis provide deep pellet mass penetration depth and increased fueling efficiency that gives DIII-D a flexible tool for transport and density control studies. 


\section{ACKNOWLEDGMENTS}

We gratefully acknowledge the support and assistance of the DIII-D Operations Group at General Atomics and the Plasma Fueling Group at ORNL. Work supported by the U.S. Department of Energy under Contracts Nos. DE-AC03-99ER54463 and DEAC05-00OR22725. 


\section{REFERENCES}

[1] S.L. Milora et al., Nucl. Fusion 35, 967 (1995).

[2] P.T. Lang et al., Phys. Rev. Lett. 79, 1487 (1997).

[3] L.R. Baylor et al., Phys. Plasmas 7, 1878 (2000).

[4] A. Geraud et al., Proc. 23rd European Conf. on Controlled Fusion and Plasma Physics, Kiev, Ukraine, Vol. 20C, Part I, p. 460 (European Physical Society, PetitLancy, Switzerland, 1996).

[5] L.R. Baylor, T.C. Jernigan, et al., J. Nucl. Mat. 457, 266 (1999).

[6] S.K. Combs et al., J. Vac. Sci. Technol. A 6(3), 1901 (1988).

[7] S.K. Combs et al., Proc. 18th IEEE/NPS Symp. on Fusion Engineering, October 25-29, 1999, San Diego, California, Vol. 1, p. 202 (Institute of Electrical and Electronics Engineers, Inc., Piscataway, New Jersey, 1999).

[8] P.B. Parks et al., Phys. Plasmas 7, 1968 (2000).

[9] P.T. Lang et al., Nucl. Fusion 36, 1531 (1996).

[10] J.R. Ferron et al., Proc. 26th European Conf. on Controlled Fusion and Plasma Physics, Maastricht, Netherlands, Vol. 23J, p. 1201 (European Physical Society, Nieuwegein, 1999).

[11] G.L. Jackson et al., Phys. Rev. Lett. 67, 3098 (1991).

[12] L.R. Baylor, T.C. Jernigan, C. Hsieh, Fusion Tech. 34, 425 (1998). 